\section{Clostridium difficile infection}

\section{Tristan Clark DTM\&H MRCP, Specialist Registrar}

Martin Wiselka MD PhD FRCP, Consultant Physician

Regional Department of Infection and Tropical Medicine, University Hospitals of Leicester NHS Trust, Leicester Royal Infirmary, Leicester

\section{Clin Med 2008;8:544-7}

Clostridium difficile is a rod-shaped, Gram-positive anaerobic bacterium which produces spores that enable it to survive in the environment for prolonged periods (Fig 1). Although first isolated in 1935, it was identified as the causal agent of antibiotic associated diarrhoea and pseudomembranous colitis only in the 1970s. C. difficile-associated disease (CDAD) has become an increasingly important nosocomial infection in the UK and throughout the western world over recent years (Fig 2). In addition to morbidity and mortality, CDAD imposes a huge economic burden on the health services.

\section{Epidemiology and pathogenesis} route; it colonises the human intestinal tract and multiplies after the resident flora has been altered by antibiotic treatment. C. difficile releases two exotoxins (A and $\mathrm{B}$ ) that bind to receptors on intestinal epithelial cells and cause secretory diarrhoea and acute inflammation.

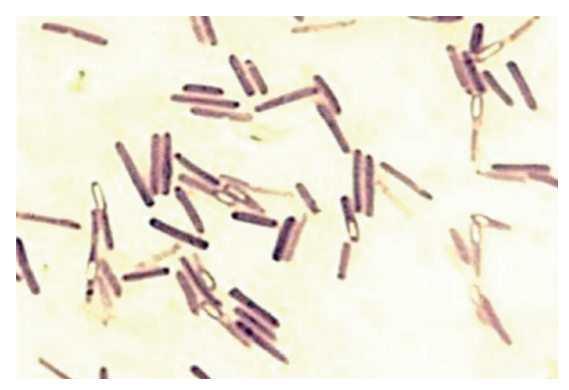

Fig 1. Clostridium difficile, showing spore formation.
C. difficile is acquired by the faeco-oral
Ulcers form on the mucosal surface, and proteins, mucous and inflammatory cells leak out forming the characteristic 'pseudomembrane' appearance in severe disease (Fig 3).

About $2 \%$ of healthy adults in the community are colonised with $C$. difficile and act as a reservoir for infection. ${ }^{1}$ Once colonised, about $60 \%$ will remain asymptomatic while the remainder develop diarrhoea. ${ }^{1}$ It is likely that the host's antibody response plays a crucial part in controlling infection. ${ }^{2}$

Nosocomial outbreaks of CDAD are common and usually caused by a single strain. Hand-washing and personal protective equipment (PPE) programmes have been shown to reduce the spread of infection. ${ }^{3}$ Recent hospital outbreaks in the USA and Europe have involved the highly virulent, quinolone-resistant strain, PCR ribotype 027 (BI), which produces up to 20 times the level of toxin $\mathrm{A}$ and $\mathrm{B}$ as other strains. ${ }^{4,5}$

\section{Risk factors for Clostridium difficile}

Antibiotic exposure is the principal risk factor for $C$. difficile acquisition and disease. All antibiotics have the potential to cause CDAD but some are associated with a higher risk (Table 1). Quinolones have been implicated in the emergence of the 027 strain. Other risk factors for colonisation and disease include nasogastric feeding and immunosuppressants. There is conflicting evidence on whether proton pump inhibitor (PPI) therapy increases the risk of $C$. difficile colonisation and CDAD, ${ }^{6}$ although PPI use is associated with a higher relapse rate.

\section{Clinical features}

C. difficile causes a spectrum of disease from asymptomatic gut colonisation through to fulminant colitis. The usual presentation of mild C. difficile disease is with acute watery diarrhoea associated with low grade fever and leukocytosis. Patients usually develop diarrhoea within seven days of starting antibiotics, but symptoms may occur on the first day of antibiotic therapy and up to three months after completing a course. In mild cases symptoms often resolve with cessation of the offending antibiotics.

More severe cases of $C$. difficile colitis present with diarrhoea, up to 15-20 stools per day, although blood in the stool is unusual. Patients have systemic toxicity, with abdominal cramps, fevers, tachycardia and marked leukocytosis. Endoscopy may reveal a patchy non-specific colitis or the classic pseudomembranes which appear as raised yellow plaques up to one $\mathrm{cm}$ in diameter (Fig 3). Complications of severe colitis include small bowel ileus or toxic megacolon (Fig 4) which may be heralded by cessation of diarrhoea. C. difficile may complicate pre-existing

\section{Key Points}

Clostridium difficile is an increasingly important nosocomial infection and the leading cause of hospital-acquired diarrhoea in the UK

C. difficile causes disease through production of toxins. Diagnosis is usually based on detection of toxin in faeces specimens

Factors associated with $C$. difficile infection include prior antibiotic therapy, prolonged hospital admission, immunosuppression, age and chronic medical conditions

Large nosocomial outbreaks of infection have occurred in the UK over recent years coinciding with the emergence of the hypervirulent ribotype 027 strain

Management includes withdrawal of the offending antibiotic(s) if possible, followed by oral metronidazole or vancomycin therapy

KEY WORDS: Clostridium difficile, diarrhoea, epidemic strain, nosocomial infection 

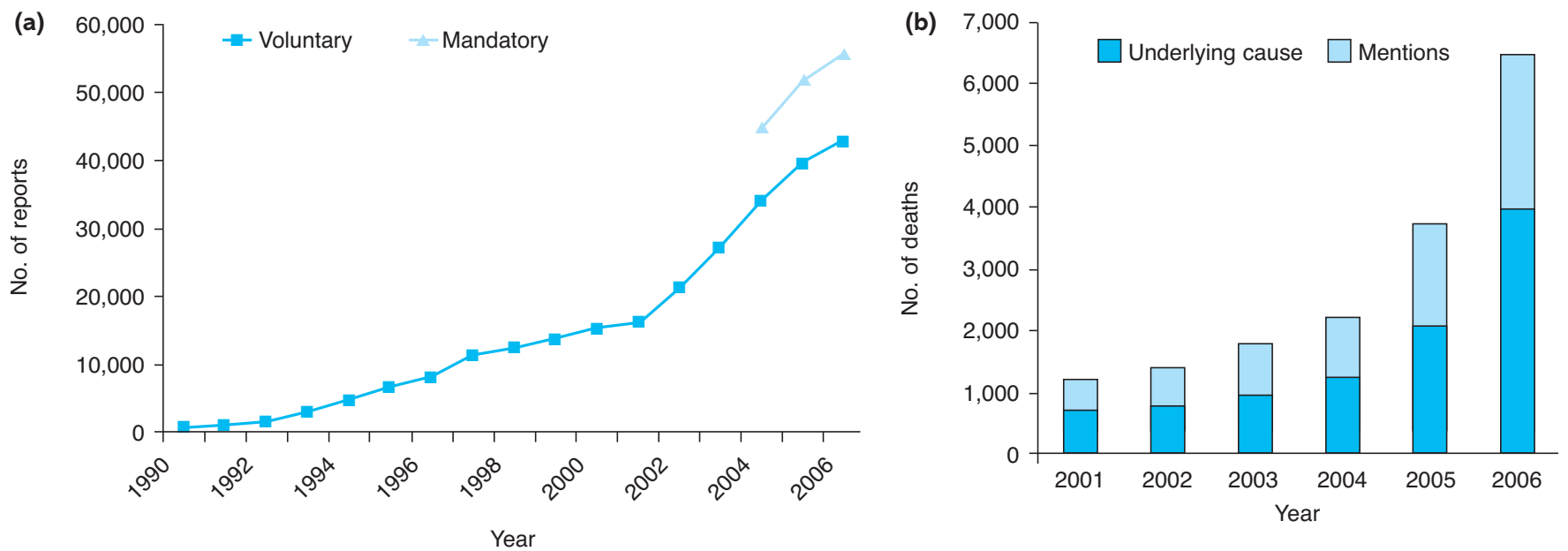

Fig 2. (a) Clostridium difficile reports in patients aged 65 years or above, England and Wales, 1990-2006; (b) C. difficileassociated deaths, England and Wales, 2001-2006. (a) Reproduced with permission from the Health Protection Agency, www.hpa.org.uk/web/HPAwebFile/HPAweb_C/1194947378214 @ Health Protection Agency; (b) Reproduced with permission from the Office for National Statistics, www.ons.gov.uk. Crown copyright material is reproduced with the permission of the Controller of OPSI. Mentions $=C$. difficile mentioned somewhere on the death certificate; underlying cause $=C$. difficile stated as a cause of death on death certificate.

inflammatory bowel disease (IBD). Treatment with steroids and immunosuppressants will worsen $\mathrm{CDAD}$, so a high index of suspicion is required in assessing patients with apparent flares, particularly if they have received antibiotics or recently been hospitalised.

\section{Diagnosis}

Any patient developing diarrhoea in hospital should have their stool tested for C. difficile.

\section{Cytotoxic bioassay}

The gold standard for diagnosis is considered to be the cytotoxic bioassay. The stool is incubated with cultured cells to look for the characteristic cytopathic

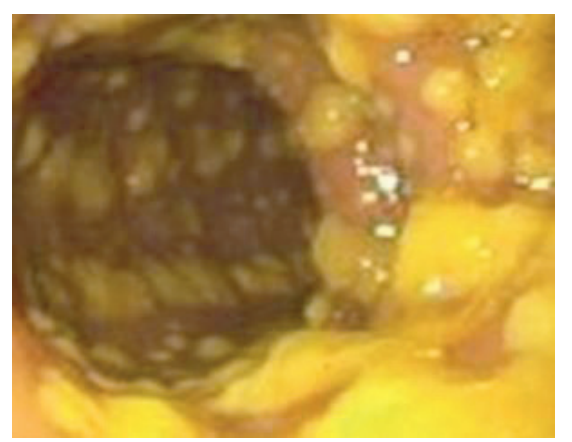

Fig 3. Sigmoidoscopic appearance of pseudomembranous colitis. effect that is abolished by neutralising antibodies to $C$. difficile toxin. It is highly sensitive and specific, but its utility is limited by expense and the time needed to complete the assay (up to three days).

\section{Rapid enzyme immunoassay}

Because of the limitations of the cytotoxic bioassay, rapid enzyme immunoassay (EIA) tests are used more widely. Commercially available kits may detect toxin A or B or both. Results can potentially be obtained within one hour, with high throughput of samples. The main disadvantage of EIA is its low sensitivity (70-90\%) so that false negative results are common. ${ }^{7}$

\section{Anaerobic stool culture}

Anaerobic stool culture is highly sensitive, but non-specific due to the existence of non-toxigenic strains of $C$. difficile that may colonise the bowel in hospitalised patients. Culture techniques are useful for epidemiological surveillance and resistance testing but usually performed only by reference laboratories.

\section{Treatment}

Offending antibiotics should be stopped wherever possible or changed to agents with a lower risk of inducing $C$. difficile. PPIs, steroids and other immunosuppressive agents should also be discontinued if possible. In symptomatic cases, empirical treatment with metronidazole or vancomycin is appropriate. It should be given if suspicion is high, even if the initial stool EIA is negative.

\section{Metronidazole}

Metronidazole is recommended for initial treatment of non-severe CDAD, based on

Table 1. Risk of Clostridium difficile infection associated with commonly used antibiotics.

\begin{tabular}{lll} 
High risk & Medium risk & Rare \\
\hline Clindamycin & Macrolides & Metronidazole \\
Second/third generation cephalosporins & Tetracyclines & Vancomycin \\
Penicillins, particularly amoxicillin/clavulanate & Aminoglycosides & \\
Quinolones (027 strain) & &
\end{tabular}


equal efficacy with oral vancomycin, cheaper cost and the theoretical risk of selecting vancomycin-resistant enterococci. $^{8}$ The standard duration of therapy is 10-14 days. Treatment should be continued for a week after cessation of other antibiotics. Repeat stool toxin testing is not warranted as the toxin test may remain positive for six weeks after resolution of symptoms.

\section{Vancomycin}

Severe disease should be treated with oral vancomycin, $125 \mathrm{mg}$ four times daily. ${ }^{8}$ Higher doses are often used, but supporting data are lacking. Intravenous (iv) metronidazole can be used in patients with intestinal ileus, but iv vancomycin has no effect on CDAD as it is not secreted into the lumen of the bowel. Intracolonic therapy with vancomycin has been used and good results reported in small case series. ${ }^{9}$

\section{Surgery}

Surgery with colectomy may be necessary in patients with severe disease refractory to therapy or in those with intestinal perforation.

\section{Relapse of Clostridium difficile- associated disease}

Relapse of CDAD is common, especially with the emerging ribotype 027 strain, occurring in $25-30 \%$ of cases with this strain. ${ }^{10}$ Some data suggest that relapse is more common in those initially treated

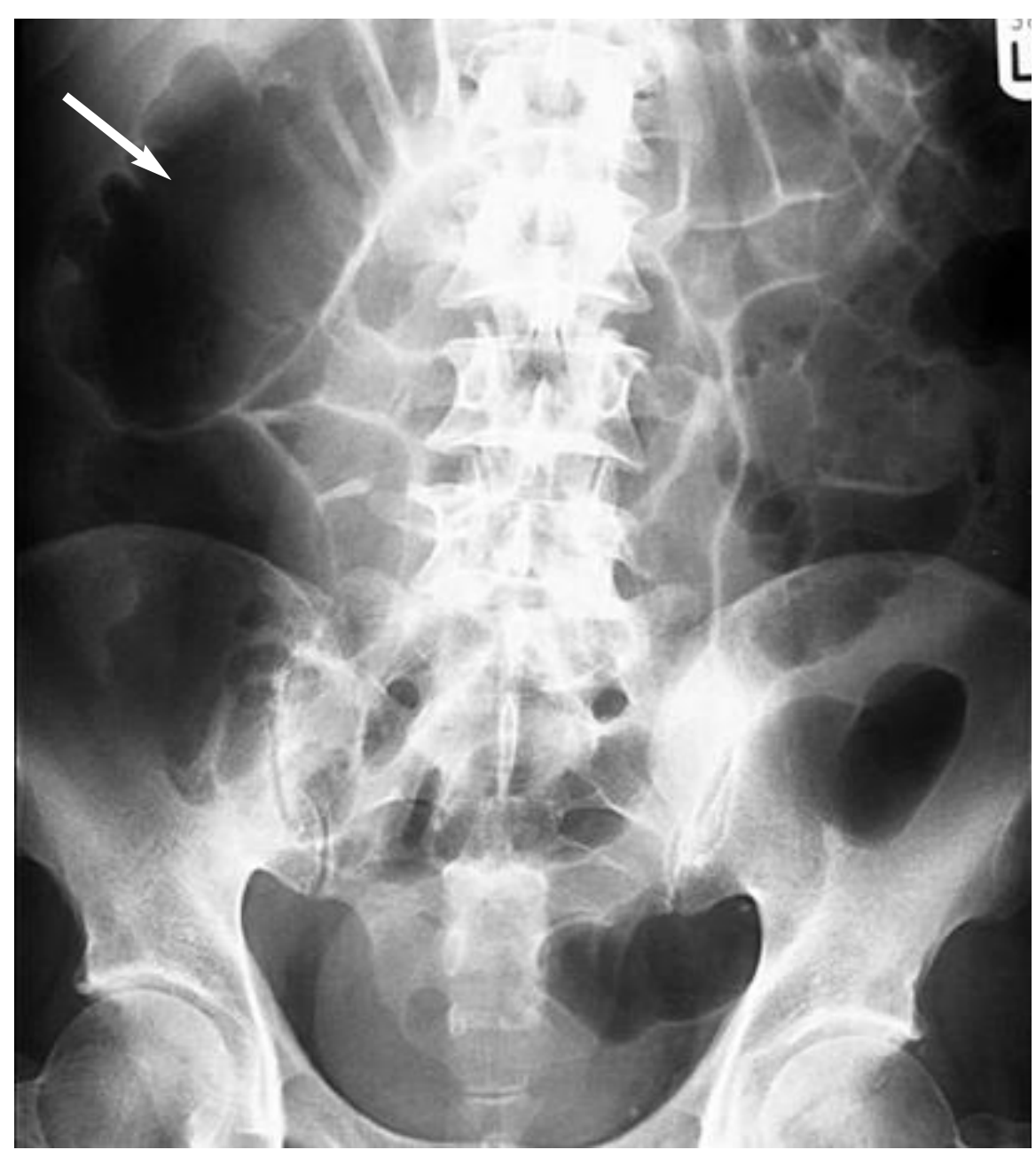

Fig 4. Toxic megacolon which may be associated with severe $C$. difficile infection. Plain abdominal radiograph showing dilated large bowel with mucosal oedema (arrow). with metronidazole rather that vancomycin, ${ }^{10}$ although resistance to metronidazole or vancomycin is not an important factor. Relapse usually occurs within two weeks of the initial episode but may arise up to three months later. Risk factors include old age, further antibiotic use, PPI therapy and residence in a nursing home. ${ }^{11}$ Relapse may represent true relapse with the same strain due to persistence of spores or may be due to reinfection with another strain. Low antibody levels against toxin A appear to correlate with risk of relapse. ${ }^{2}$

\section{Treatment of relapse}

Treatment of the first relapse of CDAD should be with metronidazole or vancomycin. ${ }^{12}$ Vancomycin is preferred if there are features of severe disease or if relapse occurs within one month of metronidazole therapy. Subsequent CDAD relapses are usually treated with tapering or pulsed vancomycin therapy. ${ }^{13}$ If the patient remains symptomatic, it is important to exclude other aetiologies, including IBD, malignancy and ischaemic colitis.

\section{Experimental therapies}

Other agents with efficacy against $C$. difficile include teicoplanin, fucidic acid, rifampicin, rifamixin and nitazoxanide, but none has been found superior to metronidazole or vancomycin. Probiotics have not been useful in initial disease but may have a role in relapsed disease. ${ }^{14}$ Other agents used include iv immunogloblin for severe CDAD cases, but its use is unsubstantiated. ${ }^{15}$ Cholestyramine has been used as a possible toxin binding agent, but there is no good trial evidence to support this approach and it may reduce the intracolonic level of vancomycin. ${ }^{16}$ The investigational agent tolevamer is a novel $C$. difficile toxin-binding resin that showed promise in initial studies but has recently been withdrawn. Research into a toxoid vaccine against C. difficile is currently underway. Faecal bacteriotherapy (the instilling of donor faeces into the bowel) seems to be successful in small case series but is not widely practised. ${ }^{17}$ 


\section{Prevention and control}

\section{Primary prevention}

Primary prevention for CDAD involves reduction of unnecessary antibiotic prescribing and the restriction of high-risk antibiotics. Both can be achieved through the use of clear hospital guidelines and antibiotic policies. ${ }^{18}$ The administration of probiotics in hospitalised patients who require antibiotics has been associated with a reduction in the subsequent incidence of antibioticassociated diarrhoea and CDAD. ${ }^{19}$

\section{Secondary prevention}

The aim of secondary prevention is to stop transmission of $C$. difficile and its spores from an index case to other hospitalised patients. This is achieved through strict isolation of patients, barrier nursing with appropriate PPE, good hand-washing procedures and environmental decontamination. Alcohol gel is ineffective against spores of $C$. difficile so hand-washing with soap and water is recommended for the care of CDAD patients. Environmental decontamination is vital as spores can survive on surfaces for several months. Solutions that reliably kill $C$. difficile spores contain sodium hypochlorite at strengths greater than 5,000 ppm.

There have recently been several highprofile outbreaks of $C$. difficile infection in UK hospitals and reporting of individual hospital infection rates was made mandatory in 2005. Many hospitals have been able to control outbreaks of infection using a comprehensive approach, combining good antibiotic stewardship, enhanced infection control measures and environmental decontamination. ${ }^{20}$

\section{References}

1 Aronsson B, Möllby R, Nord CE. Antimicrobial agents and Clostridium difficile in acute enteric disease: epidemiological data from Sweden, 1980-1982. J Infect Dis 1985;151:476-81.

2 Warny M, Vaerman JP, Avesani V, Delmée M. Human antibody response to Clostridium difficile toxin A in relation to the clinical course of infection. Infect Immun 1994;62:384-9.

3 Gerding DN, Johnson S, Peterson LR, Mulligan ME, Silvia J Jr. Clostridium difficile-associated diarrhea and colitis. Review. Infect Control Hosp Epidemiol 1995;16:459-77.

4 Blossom DB, McDonald LC. The challenges posed by reemerging Clostridium difficile infection. Clin Infect Dis 2007;45:222-7.

5 Warny M, Pepin J, Fang A et al. Toxin production by an emerging strain of Clostridium difficile associated with outbreaks of severe disease in North America and Europe. Lancet 2005;366: 1079-84.

6 Lowe DO, Mamdani MM, Kopp A, Low DE, Juurlink DN. Proton pump inhibitors and hospitalization for Clostridium difficileassociated disease: a population-based study. Clin Infect Dis 2006;43:1272-6.

7 Turgeon DK, Novicki TS, Quick J et al. Six rapid tests for direct detection of Clostridium difficile and its toxins in fecal specimens compared with the fibroblast cytotoxicity assay. J Clin Microbiol 2003;41:667-70.

8 Zar FA, Bakkanagari SR, Moorthi KM, Davis MB. A comparison of vancomycin and metronidazole for the treatment of Clostridium difficile-associated diarrhea, stratified by disease severity. Clin Infect Dis 2007;45:302-7.

9 Apisarnthanarak A, Razavi B, Mundy LM. Adjunctive intracolonic vancomycin for severe Clostridium difficile colitis: case series and review of the literature. Clin Infect Dis 2002;35:690-6.

10 Pepin J, Alary ME, Valiquette L et al. Increasing risk of relapse after treatment of Clostridium difficile colitis in Quebec, Canada. Clin Infect Dis 2005;40:1591-7.

11 Moshkowitz M, Ben-Baruch E, Kline Z et al. Risk factors for severity and relapse of pseudomembranous colitis in an elderly population. Colorectal Dis 2007;9:173-7.

12 Pépin J, Routhier S, Gagnon S, Brazeau I. Management and outcomes of a first recurrence of Clostridium difficileassociated disease in Quebec, Canada. Clin Infect Dis 2006;42:758-64.

13 McFarland LV, Elmer GW, Surawicz CM. Breaking the cycle: treatment strategies for 163 cases of recurrent Clostridium difficile disease. Am J Gastroenterol 2002;97:1769-75.

14 McFarland LV, Surawicz CM, Greenberg $\mathrm{RN}$ et al. A randomized placebo-controlled trial of Saccharomyces boulardii in combination with standard antibiotics for Clostridium difficile disease. JAMA 1994; 271:1913-8.

15 Juang P, Skledar SJ, Zgheib NK et al. Clinical outcomes of intravenous immune globulin in severe Clostridium difficileassociated diarrhea. Am J Infect Control 2007;35:131-7.

16 Taylor NS, Bartlett JG. Binding of Clostridium difficile cytotoxin and vancomycin by anion-exchange resins. J Infect Dis 1980;141:92-7.
17 Bowden TA Jr, Mansberger AR Jr, Lykins LE. Pseudomembraneous enterocolitis: mechanism for restoring floral homeostasis. Am Surg 1981;47:178-83.

18 McNulty C, Logan M, Donald IP et al. Successful control of Clostridium difficile infection in an elderly care unit through use of a restrictive antibiotic policy. J Antimicrob Chemother 1997;40:707-11.

19 Hickson M, D’Souza AL, Muthu N et al. Use of probiotic Lactobacillus preparation to prevent diarrhoea associated with antibiotics: randomised double blind placebo controlled trial. BMJ 2007;335:80.

20 Muto CA, Blank MK, Marsh JW et al. Control of an outbreak of infection with the hypervirulent Clostridium difficile BI strain in a university hospital using a comprehensive 'bundle' approach. Clin Infect Dis 2007;45:1266-73. 